\title{
Aprendizagem de Fuga após Estímulos Apetitivos Incontroláveis ${ }^{1}$
}

\author{
Angélica Capelari \\ Universidade Metodista de São Paulo \\ Maria Helena Leite Hunziker ${ }^{2}$ \\ Universidade de São Paulo
}

\begin{abstract}
RESUMO - O presente experimento teve por objetivo investigar se estímulos apetitivos não-contingentes (incontroláveis) produzem interferência na aprendizagem de fuga (desamparo aprendido). Ratos foram divididos em três grupos $(n=8)$, denominados contingente (C), não contingente (NC) e ingênuo (I). Na primeira fase (tratamento), os animais do grupo C receberam reforçamento positivo (água) sob contingência de CRF, FR5 e FR20. A cada reforço liberado a um sujeito do grupo $\mathrm{C}$, era liberada uma gota de água (não-contingente) a um sujeito do grupo NC. Os animais do grupo ingênuo (I) permaneceram no biotério. Na fase seguinte (teste), os animais foram submetidos a choques que poderiam ser desligados em função da resposta de saltar na shuttlebox. Todos os sujeitos aprenderam igualmente a resposta de fuga, independentemente do tratamento prévio recebido. Esses resultados sugerem que estímulos apetitivos incontroláveis não produzem o desamparo aprendido. Discute-se a generalização do efeito entre contextos aversivo e apetitivo.
\end{abstract}

Palavras-chave: desamparo aprendido; aprendizagem de fuga; incontrolabilidade; controle apetitivo; controle aversivo.

\section{Escape Learning after Uncontrollable Appetitive Stimuli}

\begin{abstract}
This experiment investigated whether non-contingent (uncontrollable) appetitive stimuli can produce interference on subsequent escape learning (learned helplessness). Rats were divided into three groups $(n=8)$ : contingent $(\mathrm{C})$, non-contingent (NC) or naive (I). In the first phase (treatment), the $\mathrm{C}$ animals were exposed to positive reinforcement under CRF, FR5 and FR20 schedules. Each reinforcement to the $\mathrm{C}$ animal produced a non-contingent water drop (uncontrollable stimulus) to the yoked NC animal. Animals from the I group received no treatment. In the next phase (test), all rats received electrical shocks which could be removed contingently to a jump response in a shuttlebox. All animals equally learned the escape response, regardless of the previous treatment. These results suggested that learned helplessness was not produced by uncontrollable appetitive stimuli. The generalization of the learned helplessness effect among aversive and appetitive contexts is discussed.
\end{abstract}

Key words: learned helplessness; escape learning; uncontrollability; appetitive control; aversive control.

A seleção do comportamento pelas suas conseqüências envolve uma dupla relação de controle: a do sujeito sobre seu meio ambiente - que através do seu comportamento pode modificá-lo -, e a do ambiente sobre o comportamento do sujeito, que sofre as consequiências das alterações ambientais produzidas por ele próprio (Skinner, 1981). Contudo, sabe-se que estímulos que não estão sob controle do sujeito também podem modificar seu comportamento. Diversos estudos demonstraram que indivíduos submetidos a choques elétricos incontroláveis apresentaram, posteriormente, dificuldade de aprender respostas de fuga, sendo que o mesmo não ocorreu quando os choques prévios foram controláveis. Esse efeito da incontrolabilidade dos choques tem sido denominado “desamparo aprendido" (Maier \& Seligman, 1976; Peterson, Maier \& Seligman, 1993).

1 Esse trabalho é parte da dissertação de mestrado defendida pela primeira autora, na Universidade de São Paulo, em 2002, sob a orientação da segunda autora. Ele foi parcialmente apresentado na XXX Reunião Anual de Psicologia, da Sociedade Brasileira de Psicologia, realizada em Brasília-DF em 2000, e encaminhado para a 30th Annual ABA Convention, realizada em Boston (USA), em 2004. As autoras agradecem a leitura crítica de Cristiano Valério dos Santos e o apoio financeiro do CNPq (bolsa institucional de Mestrado e bolsa Produtividade em Pesquisa).

2 Endereço: Universidade de São Paulo, Av. Prof. Mello Moraes, 1.721. São Paulo, SP, Brasil 05508-900. E-mail: hunziker@lexxa.com.br
Os trabalhos sobre desamparo aprendido utilizam, geralmente, três grupos de sujeitos, cada tríade tratada simultaneamente: um sujeito recebe choques que podem ser desligados após a emissão de uma resposta previamente determinada (grupo controlável); um segundo animal encontra-se acoplado ao primeiro, de forma que recebe os mesmos choques, mas suas respostas não modificam a duração dos mesmos, a qual depende exclusivamente do comportamento do primeiro sujeito (grupo incontrolável); o terceiro animal permanece no biotério ou na caixa experimental, sem receber choques (grupo ingênuo). Posteriormente a esse tratamento, todos os sujeitos são igualmente submetidos a uma nova contingência de fuga. $\mathrm{O}$ efeito de desamparo aprendido fica caracterizado quando apenas os sujeitos do grupo incontrolável mostram ausência de aprendizagem, ou aprendizagem mais lenta que a dos demais sujeitos, que aprendem fuga sem diferirem entre si. Tal delineamento tem a elegância de demonstrar que o desamparo aprendido não decorre dos choques em si, mas da sua incontrolabilidade (Seligman \& Maier, 1967).

Após os primeiros estudos realizados com cães (Seligman \& Maier, 1967; Overmier \& Seligman, 1967), o efeito de desamparo aprendido foi replicado com diversas espécies de animais, tais como ratos (Maier, Albin \& Testa, 1973), peixes (Padilla, Padilla, Ketterer \& Giacalone, 1970), gatos (Seward \& Humphrey, 1967), camundongos (Anisman, Catanzaro \& Remington, 1978), baratas (Brown, Howe \& 
Jones, 1990), galinhas (Rodd, Rosellini, Stock \& Gallup, 1997) e em humanos (por exemplo, Hiroto, 1974; Hiroto \& Seligman, 1975).

A interpretação mais aceita sobre o desamparo aprendido propõe que durante o tratamento com estímulos incontroláveis o sujeito aprende que as alterações dos estímulos independem de suas respostas, de forma que, posteriormente, ele tem maior dificuldade em aprender a relação R-S contida em contingências operantes às quais é exposto (Altenor, Volpicelli \& Seligman, 1979; Maier \& Seligman, 1976; Peterson \& cols., 1993).

Apesar dos estudos experimentais se centrarem em procedimentos aversivos envolvendo choques elétricos, a análise do desamparo vem sendo aplicada para diversos contextos, desde aversivos que não envolvem choque até contextos apetitivos (Peterson \& cols., 1993). Um exemplo disso é a proposta do desamparo aprendido como um modelo animal de depressão humana (Seligman, 1975). Contudo, no laboratório, essa generalidade do efeito entre contextos não é bem estabelecida, havendo resultados experimentais conflitantes. Por exemplo, ao se investigar se estímulos apetitivos incontroláveis dificultam a aprendizagem frente a novas contingências operantes, alguns estudos obtiveram baixos índices de aprendizagem (Caspy \& Lubow, 1981; Enberg, Welker, Hansen \& Thomas, 1972; Ferrándiz \& Vicente, 1997; Job, 1987; 1988; 1989; Sonoda \& Hirai, 1992; Welker, 1976; Wheatley, Welker \& Miles, 1977), enquanto outros mostraram que tal tratamento não afetou a aprendizagem futura, ou seja, não produziu desamparo (Beatty \& Maki, 1979; Calef, Metz, Atkinson, Pellerzi, Taylor \& Geller, 1984).

Conforme analisado por Hunziker (2003), essa diversidade de resultados pode ser decorrente da grande variação de procedimentos utilizados, os quais nem sempre respeitaram a conceituação básica ou a definição do efeito de desamparo aprendido. Com isso, tem-se que uma mesma denominação (desamparo) pode ser dada para efeitos que envolvem processos distintos, dificultando uma comparação direta entre os diversos estudos.

O presente experimento foi realizado com o objetivo de investigar se estímulos apetitivos incontroláveis podem produzir, posteriormente, dificuldade de aprendizagem de fuga. Para isso considerou-se crítico manter os procedimentos básicos que permitam a sua comparação com os estudos sobre desamparo realizados em contextos puramente aversivos. Como variável dependente, foi mantido o reforçamento negativo da resposta de saltar em uma caixa de dois compartimentos, contingência essa que tem se mostrado altamente sensível ao tratamento com choques incontroláveis, produzindo sistematicamente o efeito de desamparo aprendido (Hunziker, 2003). Como alteração a ser investigada nesse experimento, os choques incontroláveis foram substituídos por estímulos apetitivos (água) liberados de forma não contingente às respostas do sujeito.

\section{Método}

\section{Sujeitos}

Foram utilizados 24 ratos albinos, machos, Wistar, experimentalmente ingênuos, de aproximadamente 120 dias de idade, provenientes do Biotério da Faculdade de Psicologia da Fundação Padre Anchieta. Os sujeitos foram mantidos em gaiolas individuais por, no mínimo, uma semana antes de iniciar o experimento, com alimento (ração seca, balanceada, da marca Purina) e água sempre disponíveis. Uma semana antes do início da coleta de dados, os animais foram privados de água através do esquema de acesso à água por 6 minutos/dia, sendo que, nos dias em que houve sessão experimental, os animais receberam 3 minutos de acesso à água após a sessão. Todos os sujeitos foram pesados diariamente para controlar as suas condições de saúde. Os procedimentos experimentais foram realizados durante o período de luz do ciclo de 12 horas luz/escuro (7:00 às 19:00).

\section{Equipamento}

Foram utilizadas três caixas iguais entre si, medindo 20,0 $\mathrm{X} 23,5 \times 22,0 \mathrm{~cm}$ (profundidade, largura e altura respectivamente). Essas caixas tinham paredes laterais e o fundo feito em alumínio, parede frontal e o teto de em acrílico transparente, e o piso composto por barras cilíndricas de $0,3 \mathrm{~mm}$ de diâmetro, distando $1,3 \mathrm{~cm}$ entre si. Na parede lateral direita havia duas barras cilíndricas de latão medindo $4,0 \mathrm{~cm}$ de comprimento e $0,5 \mathrm{~cm}$ de diâmetro, eqüidistantes do centro da parede, afastadas $10,5 \mathrm{~cm}$ entre si (centro a centro), localizadas a $8,5 \mathrm{~cm}$ acima do piso. A barra direita permaneceu travada durante todas as sessões. Assim, apenas a barra esquerda podia ser pressionada pelo sujeito. Uma pressão de no mínimo 45 gf (grama-força) nessa barra produzia o acionamento de um microswitch localizado do lado externo da caixa, o qual era acompanhado de um ruído audível tipo "clique". O acionamento desse microswitch era registrado automaticamente, caracterizando a emissão de uma resposta de pressão à barra. No centro dessa parede, eqüidistante das barras e ao nível do piso, havia um bebedouro com capacidade de liberar 0,05 cc de água, que correspondia a um reforço.

As caixas permaneceram dentro de câmaras construídas em compensado e fórmica que produziram isolamento visual e acústico, sendo que um visor tipo "olho mágico" permitia a observação dos sujeitos durante as sessões. Um exaustor, localizado na parede lateral direita da câmara de isolamento, produzia renovação do ar e ruído constante. Foi utilizada como iluminação ambiente uma lâmpada de $30 \mathrm{~V}$ e $12 \mathrm{~W}$, colocada no teto da caixa de isolamento.

Uma shuttlebox também foi utilizada, medindo 50,0 X $15,5 \times 20,0 \mathrm{~cm}$ (comprimento, largura e altura), confeccionada em acrílico pintado de preto fosco, com exceção da parede frontal, que era transparente, permitindo a visualização do sujeito. A caixa era dividida em dois compartimentos, de igual tamanho, por uma parede de acrílico contendo um orifício central de $7,5 \mathrm{~cm}$ de altura e $6,0 \mathrm{~cm}$ de largura, a $8,0 \mathrm{~cm}$ acima do piso. Esse orifício possibilitava a passagem do sujeito de um compartimento ao outro da caixa. Cada compartimento tinha assoalho independente dotado de um sistema móvel que produzia sua inclinação pelo peso do sujeito. Ao ser inclinado, o piso ativava um microswitch que registrava a presença do sujeito naquele compartimento. $\mathrm{O}$ piso era composto de barras de latão cilíndricas de $0,3 \mathrm{~mm}$ de diâmetro, distando $1,3 \mathrm{~cm}$ entre si. Duas barras semelhantes 
localizavam-se na base do orifício da parede central. Essas barras, e as do piso, eram conectadas a um gerador de choques elétricos de corrente alternada (AC) com alternador de polaridades (shock scrambler), de fabricação BRS Foringer, modelo 901. Os pisos eram eletrificados separadamente, sendo que a presença do sujeito em um dos compartimentos acionava uma chave comutadora que controlava a aplicação do choque apenas nesse compartimento, de forma que o lado oposto da caixa permanecia sem eletrificação. Independentemente da localização do sujeito, as duas barras do orifício central permaneciam eletrificadas durante todo o período em que o gerador de choques estivesse acionado.

A umidade relativa do ar foi controlada através de um higrômetro localizado dentro da sala de coleta de dados. Ela foi mantida abaixo de $70 \%$ através de um desumificador (Marca Arsec Modelo 160M3-U) que era acionado sempre que esse limite era ultrapassado.

Controles e registros foram realizados por computadores que se localizavam em salas adjacentes à de coleta de dados. Na fase de tratamento, utilizou-se um computador Machintosh Color Classic com programa escrito em linguagem True Basic. Na fase de teste, utilizou-se um computador PC 386 com programa escrito em linguagem Delphi, ambos especialmente desenvolvidos para esse experimento.

\section{Procedimento}

O experimento foi composto de quatro sessões sucessivas, com intervalo de $24 \mathrm{~h}$ entre elas, divididas em duas fases: tratamento (três sessões) e teste (uma sessão). Os sujeitos, distribuídos em três grupos $(\mathrm{n}=8)$ a partir dos seus pesos corporais, foram submetidos a diferentes procedimentos na fase de tratamento. Os sujeitos do grupo contingente (C) foram submetidos, na Sessão 1, ao treino de bebedouro, seguido da modelagem da resposta de pressão à barra (por aproximações sucessivas), com 100 reforços em esquema de reforço contínuo (CRF). Na Sessão 2, o reforço foi liberado a cada cinco respostas de pressão à barra (FR5), e na Sessão 3, a cada 20 respostas emitidas (FR20). Esse aumento de razão só ocorria se o sujeito apresentasse uma taxa mínima de seis respostas por minuto em FR5; caso contrário, uma nova sessão de FR5 era realizada antes de a razão ser aumentada, acrescentando-se uma sessão ao treino. Os sujeitos do grupo não-contingente (NC) foram submetidos ao treino de bebedouro e depois acoplados aos sujeitos do grupo $\mathrm{C}$, de forma que a cada reforço recebido pelo sujeito $C$ uma gota d'água era simultaneamente liberada para seu par NC. Dessa forma, os sujeitos dos dois grupos receberam iguais quantidades de água, com a única diferença que essa apresentação era controlada pelos animais do grupo C, sendo incontrolável para os do grupo NC. O critério de encerramento dessas sessões foi a liberação de 100 reforços ou a passagem de 50 minutos, o que ocorresse primeiro. Os animais do grupo ingênuo (I) permaneceram no biotério, tendo como única manipulação o regime de privação de água. Esses sujeitos não receberam treino ao bebedouro. Nessa fase, foram registradas automaticamente as respostas de pressão à barra emitida pelos sujeitos dos grupos $\mathrm{C}$ e NC, sendo feita observação direta assistemática dos sujeitos $\mathrm{NC}$ para registro do consumo da água liberada e de outros comportamentos que poderiam se tornar freqüentes ao longo das sessões.
Posteriormente, todos os animais foram submetidos individualmente à sessão de teste, na qual foi verificada a aprendizagem de uma resposta negativamente reforçada (fuga). Essa sessão foi realizada na shuttlebox, onde foram liberados 50 choques elétricos, não sinalizados, de $1 \mathrm{~mA}$, com 10 s de duração máxima, ministrados a intervalos variáveis de $60 \mathrm{~s} \mathrm{(amplitude} \mathrm{de} \mathrm{variação} 10$ - 110 s). O choque era imediatamente interrompido caso o sujeito saltasse para o compartimento oposto (resposta de fuga). Não ocorrendo a resposta de fuga, o choque era interrompido automaticamente após $10 \mathrm{~s}$ do seu início. Cada choque correspondeu a uma tentativa, sendo o tempo decorrido entre o seu início e término registrado como a latência da resposta na tentativa. Latências de $10 \mathrm{~s}$ foram registradas como falhas. Respostas emitidas no intervalo entre choques não tiveram nenhuma conseqüência programada.

Ao término de cada sessão, foram retirados os detritos deixados pelo sujeito nas caixas experimentais, sendo as mesmas limpas com um pano umedecido em água e, em seguida, com álcool.

O Quadro 1 resume o delineamento utilizado neste estudo.

Quadro 1. Grupos experimentais compostos em função dos diferentes tratamentos recebidos antes do teste: água liberada de forma contingente (C), não contingente (NC) e ingênuo (I). Na fase de tratamento: esquema de reforçamento contínuo (CRF), razão fixa 5 (FR5) e razão fixa 20 (FR20). $\mathrm{Na}$ fase de teste: resposta (R), intervalo entre tentativas (IET)

\begin{tabular}{|c|c|c|c|c|}
\hline \multirow{3}{*}{ Grupos } & \multicolumn{3}{|c|}{ Tratamento (apetitivo) } & Teste (aversivo) \\
\hline & \multicolumn{3}{|c|}{ Resposta de Pressão à Barra } & Resposta de saltar \\
\hline & Sessão 1 & Sessão 2 & Sessão 3 & Sessão 1 \\
\hline $\mathrm{C}$ & $\begin{array}{l}\text { Modelagem } \\
\text { / CRF }\end{array}$ & FR5 & FR20 & \multirow{3}{*}{$\begin{array}{l}\text { Fuga } \\
60 \text { choques } \\
\text { Duração máx.= } 10 \mathrm{~s} \\
1,0 \mathrm{~mA} \\
\text { IET } 60 \mathrm{~s}(10-110)\end{array}$} \\
\hline $\mathrm{NC}$ & \multicolumn{3}{|l|}{$\begin{array}{l}\text { Acoplado } \\
\text { (ao grupo C) }\end{array}$} & \\
\hline I & - & - & - & \\
\hline
\end{tabular}

Os resultados foram analisados estatisticamente pelo teste ANOVA two-way para medidas repetidas, teste Tukey para comparação entre pares de grupos e teste $\mathrm{T}$ para amostras emparelhadas.

\section{Resultados}

$\mathrm{Na}$ fase de tratamento, os sujeitos que exerceram controle sobre a liberação de água (grupo C), emitindo a resposta de pressão à barra, apresentaram alta freqüência dessa resposta, enquanto que os sujeitos que receberam água não-contingente (grupo NC) praticamente não pressionaram a barra (Figura 1). A taxa de resposta do grupo $C$ foi função do esquema de reforçamento em vigor: quando o reforçamento se tornou intermitente, a taxa de respostas foi maior do que sob esquema de reforçamento contínuo (CRF). Contudo, esse aumento não foi proporcional ao valor da razão: quando a contingência de FR5 passou a FR20, houve pequena queda na taxa de respostas. Estatisticamente, as diferenças são significantes entre os grupos $(F(1,14)=140,968, p<0,001)$ e entre as sessões do grupo $\mathrm{C}(F(2,28)=72,031 ; p<0,001)$, havendo interação entre grupo e contingência $(F(2,28)=73,820 ; p<0,001)$. A comparação de grupos dois a dois mostrou que os grupos 


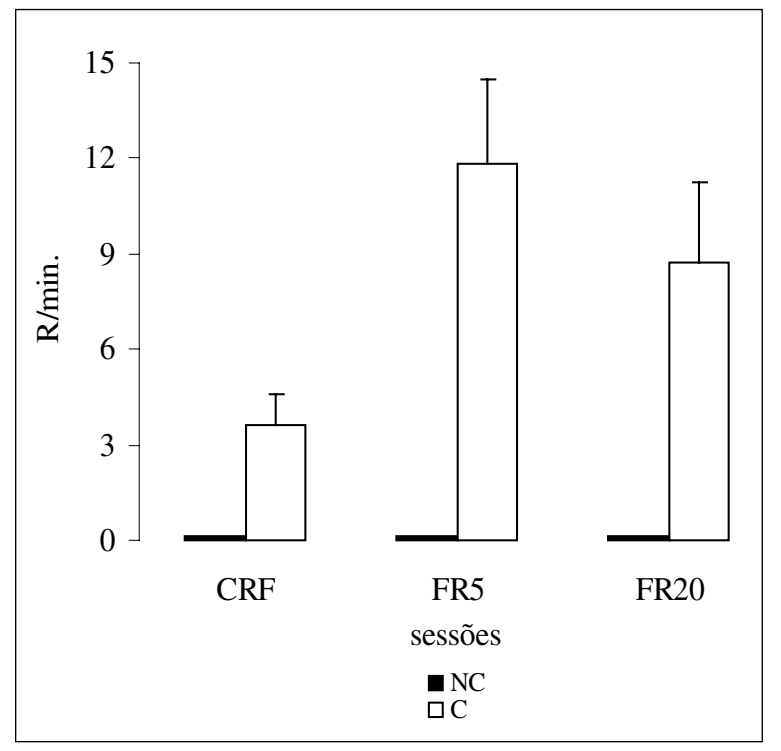

Figura 1. Taxa de respostas de pressão à barra (R/min.), apresentada pelos grupos contingente $(\mathrm{C})$ e não contingente $(\mathrm{NC})$ nas sessões de CRF, FR5 e FR20 da fase de tratamento.
$\mathrm{C}$ e NC diferiram entre si em todas as sessões $(p<0,001)$. No grupo $\mathrm{C}$, foram significantes as diferenças obtidas entre as taxas de resposta sob CRF e FR $(p<0,01)$, não havendo diferença entre as taxas de resposta emitidas em ambos os esquemas de razão.

Os resultados individuais desses sujeitos (Figura 2) mostram que todos os sujeitos $\mathrm{C}$ emitiram a resposta de pressão à barra nas três sessões de tratamento, enquanto que algumas poucas respostas foram emitidas pelos sujeitos do grupo $\mathrm{NC}$, com ocorrências que não se mantiveram através das sessões. Nesse grupo, os Sujeitos 1 e 2 apresentaram algumas poucas respostas na primeira sessão e nenhuma resposta nas sessões sucessivas; o Sujeito 3 emitiu poucas respostas apenas na segunda sessão, e o Sujeito 5 apenas na última sessão. Os demais sujeitos não emitiram essa resposta.

A observação direta dos sujeitos mostrou que todas as gotas de água liberadas foram consumidas imediatamente pelos sujeitos de ambos os grupos. No grupo NC não foram identificados comportamentos sistemáticos que antecedessem a liberação da água: durante toda a sessão, os sujeitos permaneceram próximos ao bebedouro, lambendo o mesmo logo após a liberação da gota d'água.

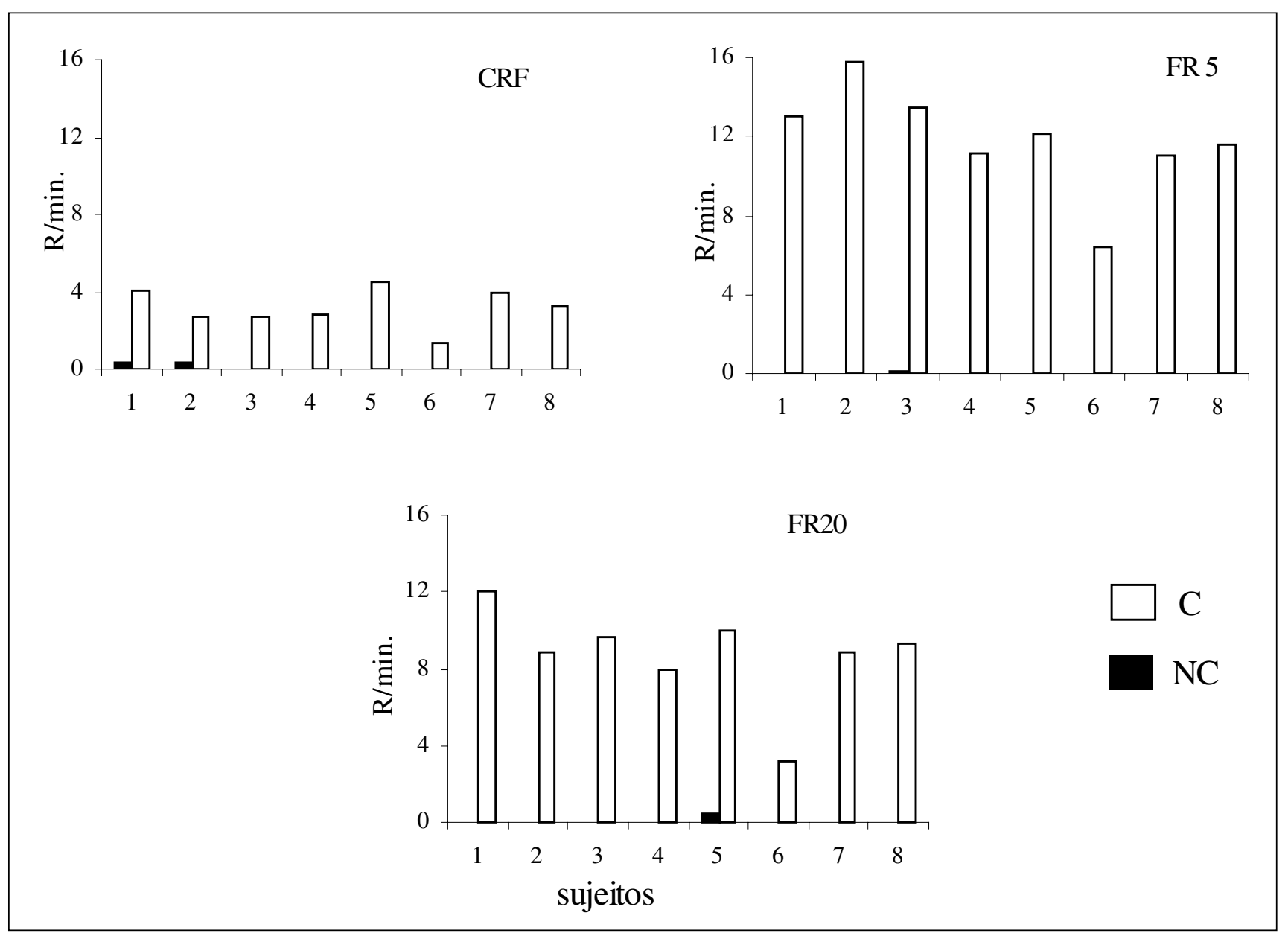

Figura 2. Taxa de respostas de pressão à barra (R/min.), apresentada individualmente pelos sujeitos dos grupos contingente (C) e não contingente (NC) nas sessões de CRF, FR5 e FR20 da fase de tratamento. 


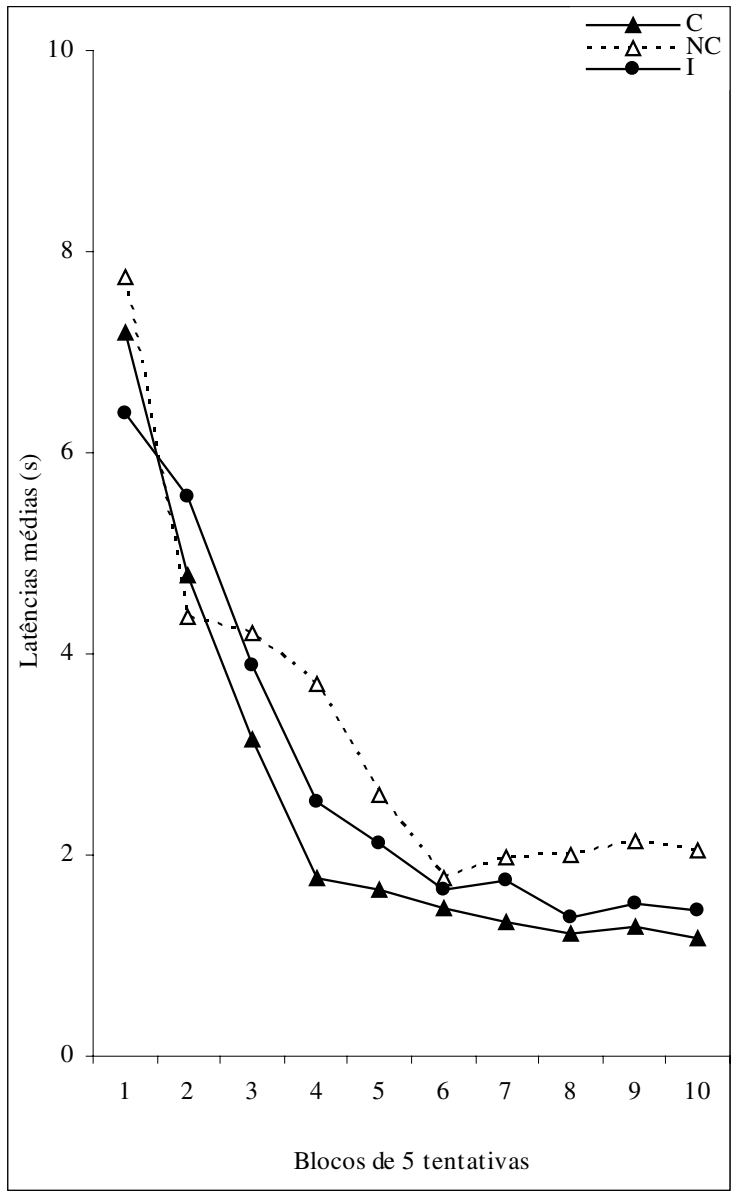

Figura 3. Latências médias (s) da resposta de saltar na shutlebox, frente a contingência de fuga, em blocos de 5 tentativas, apresentadas pelos grupos contingente (C), não contingente (NC) e ingênuo (I), na fase de teste.

Na sessão de teste, os sujeitos dos três grupos aprenderam a resposta de fuga. A Figura 3 mostra que a latência média diminuiu gradual e sistematicamente ao longo da sessão, sem distinção entre os grupos. No primeiro bloco, as latências médias foram altas, ficando entre $6 \mathrm{~s}$ e $8 \mathrm{~s}$. Nas tentativas intermediárias caíram progressivamente até um piso abaixo de $2 \mathrm{~s}$, mantendo-se estáveis nesses baixos patamares até o final da sessão. Os resultados estatísticos confirmaram que ocorreu redução das latências em função da exposição repetida ao reforçamento $(F(9,189)=56,928 ; p<0,001)$, porém sem diferença entre os grupos.

A análise do comportamento individual dos sujeitos de cada grupo confirma que todos aprenderam a emitir a resposta de fuga (Figura 4). Apesar de algumas diferenças individuais, todos terminaram as sessões com latências muito inferiores às iniciais.

Além das alterações nas latências das respostas, o reforçamento negativo também aumentou a probabilidade de ocorrência da resposta de fuga ao longo da sessão. A Tabela 1 mostra que, em todos os grupos, as falhas ocorreram concentradamente no primeiro bloco, sofrendo redução ao longo das tentativas e ficando totalmente ausentes nos três blocos finais. Essa diferença decorrente do reforçamento negativo ao longo das tentativas foi estatisticamente significante $(F$ $(9,189)=36,136 ; p<0,001)$. Embora os sujeitos do grupo NC tenham apresentado maior número de falhas que os demais, essas diferenças não foram estatisticamente significantes.

\section{Discussão}

Os resultados aqui obtidos mostram que a liberação de água, de forma não-contingente, não produziu interferência na aprendizagem de fuga posterior, ou seja, não produziu desamparo aprendido. Eles confirmam alguns estudos anteriores que não obtiveram desamparo em função da exposição prévia a estímulos apetitivos incontroláveis (Beaty \& Maki, 1979; Calef \& cols., 1984; Caspy \& Lubow, 1981; Sonoda, Takahiro \& Hirai, 1991) mas contradizem outros que relataram dificuldade de aprendizagem negativamente reforçada (Caspy, Formmer, Weiner \& Lubow, 1979; Goodkin, 1976; Sonoda \& Hirai, 1992). Esse conflito de resultados aparentemente decorre de algumas diferenças de procedimento que dificultam identificar que variáveis podem ser as responsáveis

Tabela 1. Falhas na emissão da resposta de saltar dos sujeitos (S) dos grupos contingente (C), Não-contingente (NC) e ingênuo (I), na sessão de teste. Caselas em branco significam ausência de falhas

\begin{tabular}{|c|c|c|c|c|c|c|c|c|c|c|c|c|c|c|c|c|c|c|c|c|c|c|c|c|c|c|c|}
\hline \multirow{2}{*}{$\begin{array}{l}\text { Blocos } \\
\text { blocos }\end{array}$} & \multicolumn{9}{|c|}{ Grupo C } & \multicolumn{9}{|c|}{ Grupo NC } & \multicolumn{9}{|c|}{ Grupo I } \\
\hline & S1 & S2 & S3 & S4 & S5 & S6 & S7 & S8 & $\mathbf{T}$ & S1 & $\mathbf{S 2}$ & S3 & S4 & S5 & S6 & S7 & S8 & $\mathbf{T}$ & S1 & S2 & S3 & S4 & S5 & S6 & S7 & S8 & $\mathbf{T}$ \\
\hline 1 & 3 & 3 & 2 & 4 & 1 & 2 & 1 & 3 & 19 & 4 & 2 & 5 & 1 & 2 & 5 & 3 & 1 & 23 & & 4 & 1 & 3 & 2 & 1 & 2 & 2 & 15 \\
\hline 2 & & 3 & & 2 & & 1 & & 1 & 7 & & & 3 & & & 3 & & & 6 & 2 & 2 & 1 & 3 & & 2 & 3 & & 13 \\
\hline 3 & & 2 & & & & & 3 & & 5 & & & 3 & & & 3 & & & 6 & 1 & 1 & & & & 1 & & & 3 \\
\hline 4 & & & & & & & & & 0 & & 1 & 1 & & & 3 & & & 5 & & & & & & & & & 0 \\
\hline 5 & & & & & & & & & 0 & & & & & & 1 & & & 1 & & & & 1 & & & & & 1 \\
\hline 6 & & & & & & & & & 0 & & & & & & & & & 0 & & & & & & & & & 0 \\
\hline 7 & & & & & & & & & 0 & & & & & & 1 & & & 1 & & & & & & 1 & & & 1 \\
\hline 8 & & & & & & & & & 0 & & & & & & & & & 0 & & & & & & & & & 0 \\
\hline 9 & & & & & & & & & 0 & & & & & & & & & 0 & & & & & & & & & 0 \\
\hline 10 & & & & & & & & & 0 & & & & & & & & & 0 & & & & & & & & & 0 \\
\hline TOTAL & 3 & 8 & 2 & 6 & 1 & 3 & 4 & 4 & 31 & 4 & 3 & 12 & 1 & 2 & 16 & 3 & 1 & 42 & 3 & 7 & 2 & 7 & 2 & 5 & 5 & 2 & 33 \\
\hline
\end{tabular}


grupo C
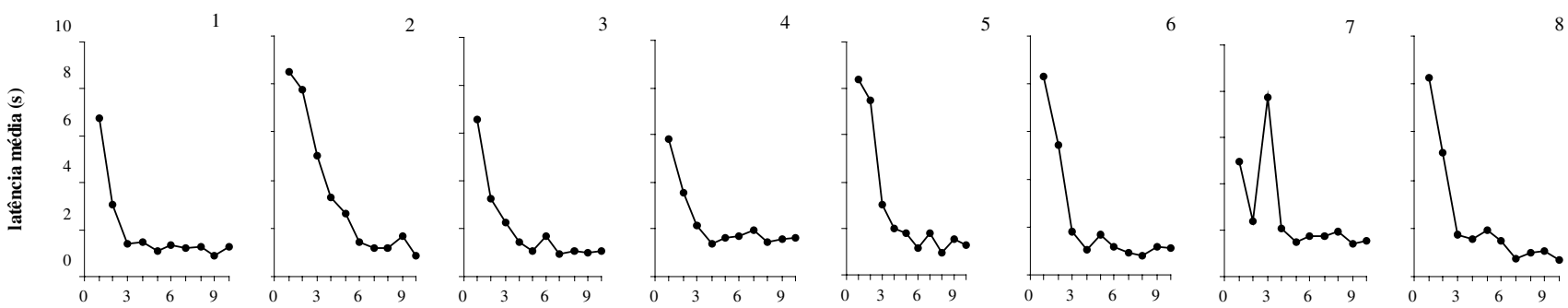

grupo NC
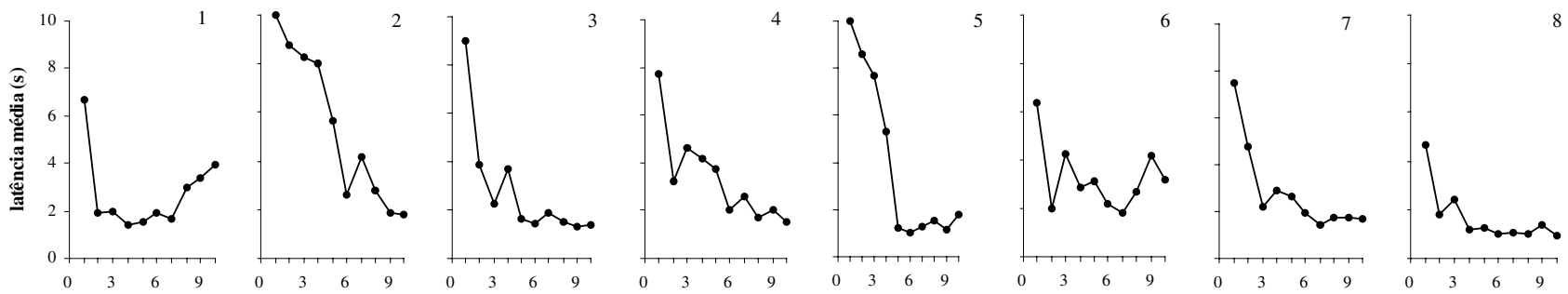

grupo I
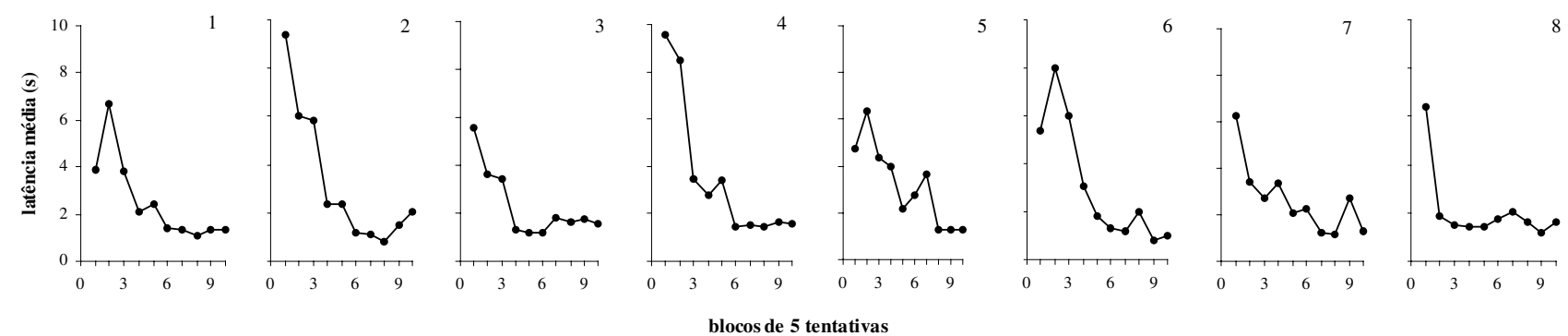

Figura 4. Latências médias (s) da resposta de saltar na shutlebox, frente a contingência de fuga, em blocos de 5 tentativas, apresentadas individualmente pelos sujeitos dos grupos contingente (C), não contingente (NC) e ingênuo (I), na fase de teste.

pelos diferentes resultados. Essas variações vão desde o tipo de resposta utilizada no teste, ao fato da contingência envolver dois ou três termos, até a forma de administrar o tratamento de incontrolabilidade. Uma diferença crítica que vem sendo apontada dentre os estudos sobre desamparo é a análise da interferência da incontrolabilidade sobre comportamentos que não têm natureza operante (Hunziker, 2003). Alguns estudos fazem isso explicitamente, utilizando no teste aprendizagens não associativas como, por exemplo, habituação (Chen \& Amsel, 1977). Contudo, mesmo outros que se propõem a analisar a interferência sobre a aprendizagem operante, eles falham em estabelecer uma contingência de reforçamento apropriada. Por exemplo, nos trabalhos de Enberg \& cols. (1972) e de Goodkin (1976), os resultados do teste mostraram um aumento das latências da resposta de fuga dos animais ingênuos no suceder das tentativas, o que indica claramente que essas respostas não estavam sob controle do reforçamento negativo (se estivessem, as latências deveriam ser reduzidas ao longo da sessão). Assim, a demonstração estatística de que o grupo exposto a choques incontroláveis foi mais lento que o grupo ingênuo não permite, nesses estudos, a conclusão de que o tratamento com a incontrolabilidade afetou um processo de aprendizagem operante (já que a contingência de teste não garantiu essa aprendizagem dentre os animais ingênuos). Ora, se o desamparo tem como variável dependen- te a aprendizagem operante, esses estudos não investigaram tal efeito, mas sim uma interferência da incontrolabilidade sobre algum aspecto do comportamento que não envolveu o controle pelas suas conseqüências.

No presente estudo, tivemos como critério manter, no teste, o mesmo procedimento que vem se mostrando eficaz na avaliação do desamparo após choques incontroláveis, ou seja, a contingência de fuga que produz aprendizagem pelos animais ingênuos e os previamente expostos a choques controláveis, e dificuldade de aprendizagem pelos tratados com choques incontroláveis. Portanto, independentemente dos demais dados da literatura, cujas diferenças de procedimento impedem uma comparação direta com o presente estudo, os atuais resultados podem ser diretamente comparados com o conjunto de experimentos que utilizam o procedimento atual, tais como Mestre e Hunziker (1996) e Yano e Hunziker (2000). Essa comparação mostra que a alteração de procedimento aqui efetuada (substituição do choque por água no tratamento com incontrolabilidade) foi crítica para os resultados opostos obtidos: a aprendizagem de fuga, com choque como reforço negativo, é dificultada pela experiência prévia com choques incontroláveis, porém, não por água incontrolável. Esses dados conflitam com a interpretação do desamparo aprendido como função apenas da incontrolabilidade do estímulo, independentemente se aversivo ou 
apetitivo (Peterson \& cols., 1993), sugerindo que embora a incontrolabilidade do estímulo possa ser apontada como uma variável crítica para o estabelecimento do desamparo aprendido, ela não garante a sua ocorrência.

Dada a implicação teórica que esses resultados têm, é importante que se identifique qual a variável se soma à incontrolabilidade na determinação do desamparo. A substituição dos choques por água, na fase de tratamento, modifica pelo menos dois aspectos que podem ser relevantes na interpretação desses resultados: a semelhança/diferença e a natureza aversiva/apetitiva dos estímulos utilizados em ambas as fases experimentais.

É possível que semelhança física entre os estímulos apresentados no tratamento e no teste seja um requisito para que a incontrolabilidade experimentada previamente interfira na aprendizagem atual. Tal semelhança entre estímulos facilita o processo de generalização, ou seja, aumenta a probabilidade de que a resposta aprendida frente a um estímulo ocorra frente a outros estímulos semelhantes a ele (Catania, 1998/1999). De fato, a maioria dos estudos sobre o desamparo aprendido com animais utiliza choques elétricos em todas as fases experimentais, ou seja, estímulos fisicamente semelhantes entre si (Peterson \& cols., 1993). Portanto, é possível que o processo de generalização de estímulos esteja presente nesses estudos, no presente experimento que utilizou água e choque nas fases de tratamento e teste, respectivamente.

Essa interpretação não conflita com a suposição de que o sujeito aprende que não tem controle sobre os choques, mas sim soma-se a ela. Se o animal aprende que determinado estímulo não é modificado pelas suas respostas, essa aprendizagem terá mais chance de se generalizar para novas situações nas quais os estímulos sejam semelhantes aos anteriores. Tal interpretação mantém a variável incontrolabilidade como crítica para a aprendizagem na primeira fase, mas sugere que há diferentes probabilidades dessa aprendizagem se generalizar para a fase de teste (desamparo) em função da semelhança ou diferença entre os estímulos utilizados.

O outro aspecto apontado como possivelmente crítico para a interpretação dos atuais resultados é a natureza aversiva/apetitiva dos estímulos utilizados. Embora alguns autores considerem que essa variável não é crítica na produção do desamparo (Peterson \& cols., 1993), ela não tem sido investigada de forma sistemática, nem tem apresentado resultados consistentes. Porém, é relevante para a compreensão do desamparo, especialmente da sua generalidade, saber se a incontrolabilidade é igualmente crítica se for relativa a estímulos aversivos ou a não aversivos. Como os estímulos aqui utilizados envolvem essas duas naturezas, mas também são fisicamente diferentes entre si, os atuais resultados não permitem uma conclusão sobre esse aspecto. Novos estudos precisam ser realizados para verificar se a natureza aversiva ou apetitiva dos estímulos, manipulados tanto na situação de tratamento como de teste, pode ser, em si, uma variável crítica para a ocorrência do desamparo aprendido. Por exemplo, seria desejável que se fizessem testes com estímulos diferentes entre si, porém ambos de natureza aversiva ou apetitiva. Isso permitiria uma primeira abordagem da relevância desses dois aspectos apontados como potencialmente críticos.

Além da sua natureza apetitiva, a água tem também como característica ser um estímulo consumatório. Pode- se argumentar que a não ocorrência do desamparo quando se utiliza estímulo apetitivo consumatório na condição de incontrolabilidade se deva ao fato de que é necessário que o sujeito emita uma determinada resposta para ter acesso a esse estímulo. A sequiência de lamber o bebedouro e ter água na boca estabelece uma relação típica operante (R-S), ou seja, de controle do sujeito sobre esse estímulo. Comparativamente, o recebimento dos choques incontroláveis nas patas depende apenas do sujeito estar com elas apoiadas sobre o piso, comportamento quase que inevitável, a menos que haja outras superfícies onde o animal possa se apoiar. Durante os choques, freqüentemente os sujeitos saltam, o que produz curtos períodos de interrupção dos choques uma vez que o animal mantém as patas afastadas do piso (por fração de segundos). Da mesma forma, não se descarta a possibilidade de que algumas posturas corporais adotadas pelo sujeito podem ter como consequiência reduzir a intensidade do choque recebido por ele. Contudo, tanto uma como outra condição que podem propiciar certo grau de controle sobre os choques, não equivalem à condição de controle observado (e necessário) com o estímulo consumatório: a resposta se aproximar do bebedouro e lambê-lo não é uma possibilidade, mas uma condição necessária para que a água atinja o sujeito. Portanto, nas sessões de choques incontroláveis é possível que exista algum pequeno grau de controle sobre a interrupção ou a intensidade do choque, porém nas sessões de água incontrolável é necessário que o animal emita determinadas respostas para que o estímulo chegue até ele. O quanto essa diferença no grau de controle possível durante o tratamento se relaciona aos resultados obtidos, é ainda uma questão a ser investigada, talvez com reforços positivos que não sejam consumatórios.

Contudo, deve-se atentar para o fato de que esse controle do sujeito acima discutido se restringe ao consumo da água, mas não à sua liberação: o procedimento adotado estabelece que o aparecimento da água na caixa experimental não é contingente a qualquer resposta. Além disso, dado o treino de bebedouro inicial, podemos considerar que o som do bebedouro e a visão da gota d'água são estímulos que adquirem propriedades reforçadoras condicionadas. Portanto, o som do bebedouro e a visão da água são estímulos incontroláveis para esse sujeito. Assim, apesar das limitações apontadas a respeito do controle sobre o consumo da água, talvez existam mais similaridades do que diferenças entre os arranjos de choque e água incontroláveis.

A liberação da água de forma não-contingente aparentemente não estabeleceu reforçamento acidental da resposta de pressão à barra (Skinner, 1948). Isso pode ser analisado em função do fato de que na condição de incontrolabilidade essa resposta teve freqüência quase nula, sem um padrão constante, reduzindo sua emissão ao longo das sessões. Ao contrário, quando a liberação da água foi contingente a essa resposta, a sua frequiência foi alta, dependente do esquema de reforçamento em vigor: maior freqüência frente ao reforçamento intermitente, em comparação ao CRF, conforme previsto na literatura (Catania, 1998/1999). A leve queda obtida durante o FR20, comparativamente ao FR5, provavelmente ocorreu em função da distensão da razão, efeito esse produzido pela passagem abrupta da razão 5 diretamente para razão 20. 
A seleção da resposta de permanecer próximo ao bebedouro, ocorrida na fase de treino, não pode ser considerada uma resposta supersticiosa uma vez que ela é condição necessária para que o estímulo manipulado atinja o sujeito. É crítico que o sujeito tenha acesso à gota d'água logo após a sua liberação não-contingente, como forma de tornar esse procedimento comparável aos que utilizam choques incontroláveis, pois o sujeito recebe esses choques imediatamente após a sua liberação. Pode-se considerar que tanto a resposta de permanecer próximo do bebedouro, no atual experimento, como a de permanecer pouco ativo durante os choques incontroláveis, relatada em diversos estudos (Maier \& Seligman, 1976; Seligman \& Maier, 1967), são respostas adaptativas que reduzem o "custo" frente a essas condições, embora não caracterizem um controle sobre a ocorrência ou duração do estímulo manipulado: a primeira apenas facilita o acesso rápido à água e a outra evita desperdiço de energia, por movimentação física não funcional, durante os choques incontroláveis.

Portando, nossos resultados fortalecem a suposição de que a incontrolabilidade dos estímulos não é uma variável suficiente para que se produza o efeito de desamparo aprendido. Novos estudos precisam ser realizados para investigar a relevância da natureza aversiva ou apetitiva dos estímulos, bem como da similaridade dos estímulos utilizados nas diferentes fases experimentais. Se confirmadas essas variáveis como críticas para a ocorrência do desamparo, a generalidade desse efeito, inicialmente considerada muito ampla (Seligman, 1975), pode sofrer restrições em função de um conjunto de variáveis e não apenas a incontrolabilidade de estímulos.

\section{Referências}

Altenor, A., Volpicelli, J. R. \& Seligman, M. E. P. (1979). Debilitated shock escape is produced by short and long duration inescapable shock: Learned helplessness vs. learned inactivity. Bulletin of Psychonomic Society, 14, 337-339.

Anisman, H. C. D. \& Remington, G. (1978). Escape performance deficits following exposure to inescapable shock: deficits in motor response maintenance. Journal of Experimental Psychology: Animal Behavior Processes, 4, 197-218.

Beatty W. W. \& Maki, W. S. (1979). Acquisition of instrumental responding following non-contingent reinforcement: failure to observe "learned laziness" in rats. Bulletin of the Psychonomic Society, 13(4), 268-271.

Brown, G. E., Howe, A. R. \& Jones, T. E. (1990). Immunization against learned helplessness in the cockroach (Periplaneta americana). Psychological Reports, 67, 623-640.

Calef, R. S., Metz, R. A., Atkinson, T. L., Pellerzi, R. C., Taylor, K. S. \& Geller, E. S. (1984). Acquisition of running in straight alley following experience with response - independent food. Bulletin of the Psychonomic Society, 22(11), 67-69.

Caspy, T., Frommer, R., Weiner, I. \& Lubow, R. E. (1979). Generality of US pre-exposure effects: effect of shock or food pre-exposure on water escape. Bulletin of the Psychonomic Society. 14(1), 15-18.

Caspy, T. \& Lubow, R. E. (1981). Generality of US pre-exposure effects: transfer from food to shock or shock to food with and without the same response requirements. Animal Learning and Behavior, 9(4), 524-532.
Catania, A. C. (1999). Aprendizagem: Comportamento, linguagem e cognição. Porto Alegre: Artes Médicas.

Chen, J. \& Amsel, A. (1977). Prolonged, unsignaled, inescapable shocks increase persistance in subsequent appetitive instrumental learning Animal Learning and Behavior, 5, 377-385.

Enberg, L. A., Welker, R. L., Hansen, G. \& Thomas, D. R. (1972). Acquisition of key pecking via auto shaping as a function to prior experience: "Learned laziness"? Science, 178, 1.002-1.004.

Ferrándiz, P. \& Vicente, F. (1997). The conditioned attention theory and bifactorial theory on the learned helplessness syndrome in appetitive contexts. International Journal of Psychology, 32(6), 399-408.

Goodkin, F. (1976). Rats learn the relationship between responding and environmental events: An expansion of the learned helplessness hypothesis. Learning and Motivation, 7, 382393.

Hiroto, D. S. (1974). Locus of control and learned helplessness. Journal of Experimental Psychology, 102, 187-193.

Hiroto, D. S. \& Seligman, M. E. P. (1975). Generality of learned helplessness in man. Journal of Personality and Social Psychology, 31, 311-327.

Hunziker, M. H. L. (2003). Desamparo aprendido. Tese de LivreDocência, Universidade de São Paulo, São Paulo.

Job, R. F. S. (1987). Learned helplessness in an appetitive discrete trial T-maze discrimination test. Animal Learning and Behavior, 15(3), 342-346.

Job, R. F. S. (1988). Interference and facilitation produced by noncontingent reinforcement in the appetitive situation. Animal Learning and Behavior, 16(4), 451-460.

Job, R. F. S. (1989). A test of proposed mechanism underlying the interference effect produced by non-contingent food presentations. Learning and Motivation, 20, 153-177.

Maier, S. F. \& Seligman, M. E. P. (1976). Helplessness: Theory and evidence. Journal of Experimental Psychology: General, 105(19), 3-46.

Maier, S. F., Albin, R. W. \& Testa, T. J. (1973). Failure to learn to escape in rats previously exposed to inescapable shock depends on nature of escape response. Journal of Comparative and Physiological Psychology, 85, 581-592.

Mestre, M. B. A. \& Hunziker, M. L. (1996). O desamparo aprendido, em ratos adultos, como função de experiências aversivas incontroláveis na infância. Tuiuti: Ciência e Cultura, 6(2), 25-47.

Overmier, J. B. \& Seligman, M. E. P. (1967). Effects of inescapable shock upon subsequent escape and avoidance learning. Journal of Comparative and Physiological Psychology, 63, 23-33.

Padilla, A. M., Padilla, C., Ketterer, T. \& Giacalone, D. (1970). Inescapable shocks an subsequent escape avoidance conditioning in goldfish, carassius aurotus. Psychonomic Science, 20, 295-296.

Peterson, C., Maier, S. F. \& Seligman, M. E. P. (1993). Learned Helplessness. A theory for the age of personal control. Nova York: Oxford University Press.

Rodd, Z. A., Rosellini, R. A., Stock, H. S. \& Gallup, G. G. Jr. (1997). Learned helplessness in chickens (Gallus gallus): Evidence for attentional bias. Learning and Motivation, 28, 43-55.

Seligman, M. E. P. \& Maier, S. F. (1967). Failure to escape traumatic shock Journal of Abnormal Psychology, 74(1), 1-9.

Seligman, M. E. P. (1975). Heplessness: On depression, development, and death. San Francisco: W. H. Freeman. 
Seward, J. P. \& Humphrey, G. L. (1967). Avoidance learning as a function of pre training in the cat. Journal of Comparative and Physiological Psychology, 63, 338-341.

Skinner, B. F. (1948). "Superstition" in the pigeon. Journal of Experimental Psychology, 38, 168-177.

Skinner, B. F. (1981). Selection by consequences. Science, 213, 501-504.

Sonoda, A. \& Hirai, H. (1992). The role of predictability in preventing escapes deficits following loss of control over. Animal Learning and Behavior, 20(4), 427-430.

Sonoda, A., Takahiro, O. \& Hirai, H. (1991). Loss of controllability in appetitive situations intereferes with subsequent learning in aversive situations Animal Learning and Behavior, 19(3), 270-275.

Welker, R. L. (1976). Acquisition of a free-operant-appetitive response in pigeons as a function of prior experience with response. Learning and motivation, 7, 394-405.
Wheatley, K. L., Welker, R. L. \& Miles, R. C. (1977). Acquisition of a bar pressing in rats following experience with responseindependent food. Animal Learning and Behavior, 5(3), 263242.

Yano, Y. \& Hunziker, M. H. L. (2000). Desamparo aprendido e "imunização" com diferentes respostas de fuga. Acta Comportamentalia, 8(2), 143-166.

Recebido em 22.06.2004 Primeira decisão editorial em 31.01.2005 Versão final em 28.02.2005 Aceito em 09.04.2005 